INRA Prod. Anim., 2008, 21 (1), 95-106

\title{
Qualité des produits : modulation par l'alimentation des animaux de la composition en acides gras du lait et de la viande
}

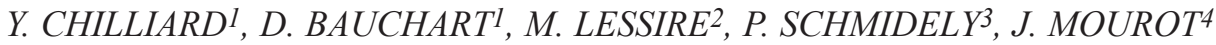

${ }^{1}$ INRA, UR1213 Herbivores, F-63122 Saint-Genès Champanelle, France

2 INRA, UR83 Recherches Avicoles, F-37380 Nouzilly, France

3 INRA, AgroParisTech, UMR791 Physiologie de la Nutrition et de l'Alimentation, F-75231 Paris, France

${ }^{4}$ INRA, Agrocampus, UMR1079 Systèmes d'Elevage, Nutrition Animale et Humaine, F-35590 Saint-Gilles, France

Courriel :yves.chilliard@clermont.inra.fr

La composition en acides gras du lait et de la viande est une composante importante de leur qualité nutritionnelle pour l'homme. Elle est fortement modulable à court terme par l'alimentation des animaux d'élevage avec des différences de réponse marquées selon les espèces.

L'alimentation joue un rôle essentiel pour la santé humaine et notamment dans le développement de maladies chroniques telles que les cancers, maladies cardiovasculaires, résistance à l'insuline et obésité (Darnton-Hill et al 2004, AFSSA 2005, Shingfield et al 2008). Une meilleure compréhension de ses effets nécessite de disposer de connaissances précises sur la qualité nutritionnelle des aliments. Celle des produits animaux dépend à la fois de leur caractéristiques biochimiques (teneurs en macro- et micro-nutriments, voire en divers contaminants) et microbiologiques (présence éventuelle de micro-organismes probiotiques ou pathogènes). Les produits animaux représentaient en 1999 plus de $80 \%$ de la consommation totale $(90 \mathrm{~g} / \mathrm{j})$ de lipides des adultes en France, avec parmi les sources identifiées $28 ; 12 ; 5 ; 3$ et $0,4 \mathrm{~g} / \mathrm{j}$ pour les produits laitiers, et les viandes de porc, de bovin, de volailles et de lapin, respectivement, auxquels il faut ajouter $5 \mathrm{~g} / \mathrm{j}$ pour les œufs, le poisson et la viande de mouton et $21 \mathrm{~g} / \mathrm{j}$ de lipides animaux d'origine non identi- fiée (enquête INCA, Volatier 2000). Ces produits sont souvent considérés, lorsqu'ils sont consommés en excès, comme des facteurs de risque pour les maladies chroniques, notamment en raison de leur richesse en Acides Gras (AG) saturés (12:0 à 16:0 en particulier) ou insaturés de structure trans (cf. encadré p. 96). Toutefois ces produits contiennent également des $\mathrm{AG}$ polyinsaturés (AGPI) de la série n-3, considérés comme bénéfiques (ANC 2001) ${ }^{1}$ ainsi que des AG «mineurs» des produits de ruminants (résultant directement ou indirectement des produits des biohydrogénations ruminales) qui ont des propriétés potentiellement anticancéreuses et/ou antiathérogènes, comme l'isomère conjugué de l'acide linoléique (CLA) 9c,11t-18:2 (ou acide ruménique) ou certains $\mathrm{AG}$ ramifiés (Jensen 2002, De La Torre et al 2005, Corino et al 2006). Par ailleurs, les effets potentiellement positifs ou négatifs des AG trans naturellement présents dans les produits de ruminants, par opposition à ceux obtenus industriellement par hydrogénation d'huiles végétales, restent encore très mal connus (Roy et al 2007, Shingfield et al 2008). Enfin, alors que certains préconisent de modifier la composition en matières premières des rations afin de les rééquilibrer, d'autres considèrent qu'une modification limitée de composition en $A G$ des produits animaux peut contribuer à modifier largement, sans changer les habitudes des consommateurs, l'apport alimentaire quotidien en ces $\mathrm{AG}$ en raison de la part importante de ces produits dans certaines rations couramment consommées.

L'objectif de modifier la composition en $A G$ des produits animaux est donc devenu prioritaire dans les recherches de l'INRA, en particulier depuis la fin des années $90^{2}$. Bien que des approches de génétique animale et de technologie des produits animaux soient possibles, une stratégie potentiellement efficace, rapide, réversible et économique est de jouer sur des modifications de composition des rations des animaux de rente. Les Départements Elevage et Nutrition des Animaux (ENA) puis Physiologie

\footnotetext{
${ }^{1}$ Le rôle important des AGPI n-3 vis-à-vis des maladies cardiovasculaires et du développement du cerveau a été mis en évidence à l'INRA dès 1970 à Jouy-en-Josas par G. Durand et collaborateurs (Durand et al 1979, Bourre et al 1984). L'intérêt pour les AGPI n-3 n'est donc pas nouveau, mais le développement des recherches autour de ces AG a été accentué après la publication des Apports Nutritionnels Conseillés par l'Agence Française de Sécurité Sanitaire des Aliments (AFSSA) (ANC 2001). Les ANC préconisent dans l'alimentation humaine un rapport 18:2 n-6/18:3 n-3 d'une valeur de 5 alors qu'il est actuellement compris entre 15 et 30. Il faut donc baisser la consommation d'AGPI n-6 et augmenter celle des n-3. La valeur proposée pour le 18:3 n-3 est de 2 g/j alors que la consommation actuelle est estimée à $850 \mathrm{mg} / \mathrm{j}$. L'AFSSA considère donc que tout nouveau vecteur alimentaire qui apportera des AGPI n-3 est à prendre en considération.

2 En outre, fin 2005, l'AFSSA a mis en place un groupe de travail, animé par P. Schmidely et auquel participent les auteurs de cet article, sur l'impact des pratiques en alimentation animale sur la composition en AG des produits animaux.
} 


\section{Nomenclature des acides gras}

Les AG sont décrits en indiquant le nombre de carbones et de doubles liaisons, le numéro du $1^{\mathrm{er}}$ carbone de chaque double liaison, compté à partir de l'extrémité carboxylique $(\mathrm{COOH})$ (numérotation en «delta»), et enfin la configuration spatiale de la double liaison : cis -c- ou trans -t- (selon que les deux parties de la chaîne carbonée sont situées du même coté ou de part et d'autre, respectivement, de la double liaison). Par exemple, le $11 \mathrm{t} 15 \mathrm{c}-18: 2$ possède 18 atomes de carbone et 2 doubles liaisons en positions delta 11 (configuration trans) et delta 15 (configuration cis).Toutefois, dans le cas des acides linoléique (18:2 n-6) et alpha-linolénique (18:3 n-3) on peut indiquer seulement, pour simplifier, le nombre de carbones, le nombre de doubles liaisons cis et la position de la 1 ère d'entre elles à partir de l'extrémité méthylique $(\mathrm{CH} 3)$ (il y a identité entre les dénominations n-3 et oméga 3, ou n-6 et oméga 6 , respectivement). Enfin, un AG est dit «conjugué» lorsque les doubles liaisons sont distantes de deux carbones (au lieu de trois habituellement).

Animale et Systèmes d'Elevage (PHASE) ont donc consacré des moyens relativement importants à leur étude durant la dernière décennie.

\section{1 / Modulation par l'ali- mentation de la composi- tion en acides gras des lipi- des du lait}

L'importance quantitative de la consommation de matières grasses laitières (cf. ci-dessus), et leur teneur élevée en AG saturés ou insaturés de structure trans, en font le principal vecteur de la consommation de ces AG. L'alimentation des vaches détermine l'essentiel des variations du profil des $\mathrm{AG}$ du lait sur le terrain (Lucas et al 2006, Ferlay et al 2008). Les variations liées à l'animal [race (Palmquist et al 1993, Ferlay et al 2006) ; stade de lactation, en liaison avec le bilan énergétique négatif après vêlage (Chilliard et al 1991)] sont de moindre importance. Les effets de la technologie laitière sur la composition en AG des produits sont minimes par rapport à ceux de l'alimentation des animaux (Chilliard et al 2006, Lucas et al 2006).

La supplémentation lipidique des rations a été utilisée depuis des décennies en recherche, et dans une certaine mesure en élevage, pour modifier les performances et le métabolisme énergétique des vaches laitières (Chilliard 1993) et/ou la composition en AG du lait (Chilliard et al 2007). Toutefois la part des suppléments lipidiques dans l'alimentation des vaches laitières reste modeste en pratique, et ce sont les variations de la nature et des proportions respectives des fourrages (herbe pâturée notamment) et des aliments concentrés qui déterminent les variations de composition en $\mathrm{AG}$ des laits collectés par l'industrie. Les tentatives pour modifier le pourcentage d'une catégorie d'AG se traduisent par des modifications simultanées d'autres AG, qui peuvent être considérés comme favorables ou défavorables à la santé humaine. Ainsi, les régimes qui diminuent la teneur en $\mathrm{AG}$ saturés et accroissent les AGPI ou le CLA entraînent généralement une augmentation des AG trans.

\section{1 / Effet de la nature des four- rages consommés}

Les teneurs de l'herbe en AG et en 18:3 n-3 varient rapidement en début de saison, expliquant que le lait soit plus riche en 18:0, 9c-18:1, 11t-18:1, 9c,11t$18: 2$ et $18: 3$ n-3, trois semaines après que six semaines après la mise à l'herbe (tableau 1) du fait de la diminution de la teneur de 1'herbe en 18:3 n-3 et que la biohydrogénation ruminale de cet $\mathrm{AG}$ en $11 \mathrm{t}-18: 1$ et $18: 0$ peut permettre la synthèse mammaire de $9 \mathrm{c}, 11 \mathrm{t}-18: 2$ et de $9 \mathrm{c}$ 18:1 (Chilliard et al 2007).

Par rapport à des rations mixtes (ensilage d'herbe ou de maïs, et 20 à $45 \%$ de concentrés), la consommation exclusive de pâturage permet d'augmenter les concentrations dans le lait de 18:0 (+ 2 points), 18:1 ( +8 points) et 18:3 (qui passe de $0,3-0,5$ à $1,4 \%$ des $A G$ totaux, et pourrait donc représenter près de $20 \%$ des ANC pour une consommation de $28 \mathrm{~g}$ par jour de matières grasses laitières), tout en diminuant celles de 10:0 à 16:0 (- 13 points ; Chilliard et al 2001). Ces données ont été confirmées et précisées récemment (Ferlay et al 2006, Couvreur et al 2007). L'ensilage de maïs est pauvre en $18: 3 \mathrm{n}-3$, et riche en 18:2 n-6 et en 9c18:1. Ceci explique qu'il augmente fortement le rapport n-6/n-3 du lait par rapport à l'ensilage d'herbe (Chilliard et al 2001), sans modifier le 9c,11t18:2 (Chilliard et al 2007) ou en l'augmentant légèrement, ainsi que le $11 \mathrm{t}$ 18:1 et le 9c,13t-18:2 (Ferlay et al 2006).
En travaillant sur des laits de tournées de ramassage, Ferlay et al (2008) ont montré que le pâturage de prairie permanente de demi-montagne, comparé à des régimes hivernaux de plaine riches en ensilage de maïs, diminue dans le lait le 16:0 (- 9,5 points) et augmente les monoinsaturés trans à $16 \mathrm{C}$ et $18 \mathrm{C}$ ( $+3,1$ points), le 9c,11t-18:2 $(+1,2$ point $)$, le $18: 3$ n-3 ( $+0,5$ point $)$ et l'EPA (qui passe de 0,04 à $0,08 \%$ des AGT). Des gradients de richesse en ces mêmes AG sont observés, dans cet ordre, entre alpage, prairie permanente, prairie temporaire, ensilage d'herbe, foin et ensilage de maïs (Lucas et al 2006). Par ailleurs, des foins provenant d'une herbe de qualité et séchés en grange, peuvent avoir des teneurs élevées en $A G$ et en 18:3n-3, permettant la production d'un lait plus riche en 18:3 n-3 que le pâturage, et plus riche en 11t-18:1 et 9c,11t-18:2 que 1'ensilage d'herbe (tableau 1).

En conclusion, les rations à base d'herbe, pâturée ou conservée dans de bonnes conditions, modifient le profil des AG du lait dans un sens potentiellement favorable, comparées aux rations riches en concentrés et/ou en ensilage de maïs.

\section{2 / Effet d'apports de lipides alimentaires}

Les effets des suppléments lipidiques, et les relations dose-réponse, ont été évalués soit en compilant les résultats extraits d'une base de données bibliographiques (270 lots de vaches supplémentées et 140 lots de vaches non supplémentées, Glasser et al non publié), soit en utilisant des résultats d'études spécifiques (Chilliard et al 2007), de façon à évaluer certains effets «toutes choses égales par ailleurs», ce qui n'est pas possible inter-essais en raison du nombre limité de publications décrivant précisément les isomères des AG trans et/ou polyinsaturés.

Le potentiel de diminution des $\mathrm{AG}$ saturés, en particulier de 10:0 à 16:0, est considérable. Par exemple, ces derniers diminuent de 56 à $29 \%$ des $\mathrm{AG}$ totaux après addition de $5 \%$ d'huile de lin à une ration à base de foin (Roy et al 2006). Inversement, les savons de calcium d'huile de palme, qui sont les plus utilisés actuellement en élevage laitier sur le terrain, accroissent la teneur en $16: 0(+21 \mathrm{mg} / \mathrm{g}$ pour un apport moyen de $770 \mathrm{~g} / \mathrm{j}$, Chilliard et al 1993).

La supplémentation en suifs (graisses corporelles de ruminants, riches en 
Tableau 1. Effets de la nature des fourrages sur la composition en acides gras du lait de vache (Ferlay et al 2006).

\begin{tabular}{|c|c|c|c|c|c|c|c|}
\hline \multirow{2}{*}{$\begin{array}{c}\text { Acides gras } \\
\text { (g/100 g } \\
\text { d'AG totaux) }\end{array}$} & \multicolumn{7}{|c|}{ Régime $^{1}$} \\
\cline { 2 - 8 } & Concentré & $\begin{array}{c}\text { Ensilage } \\
\text { de maïs }\end{array}$ & $\begin{array}{c}\text { Ensilage } \\
\text { d'herbe }\end{array}$ & $\begin{array}{c}\text { Foin de } \\
\text { raygrass }\end{array}$ & $\begin{array}{c}\text { Foin de } \\
\text { prairie } \\
\text { naturelle }\end{array}$ & $\begin{array}{c}\text { Pâturage } \\
\text { S3 }\end{array}$ & $\begin{array}{c}\text { Pâturage } \\
\text { S6 }\end{array}$ \\
\hline $10: 0+12: 0+14: 0$ & 21,9 & 19,3 & 18,7 & 19,6 & 19,4 & 14,8 & 16,5 \\
\hline $16: 0$ & 33,5 & 31,0 & 32,1 & 30,2 & 30,0 & 22,9 & 29,6 \\
\hline $18: 0$ & 6,7 & 7,9 & 7,9 & 8,2 & 9,0 & 11,4 & 9,2 \\
\hline cis9-18:1 & 14,1 & 16,7 & 16,0 & 15,4 & 17,4 & 24,1 & 21,8 \\
\hline trans11-18:1 & 0,6 & 1,0 & 0,9 & 1,8 & 1,3 & 3,7 & 1,8 \\
\hline $18: 2$ n-6 & 1,8 & 1,5 & 1,1 & 1,0 & 1,2 & 1,1 & 1,2 \\
\hline $18: 3$ n-3 & 0,5 & 0,2 & 0,9 & 1,0 & 1,3 & 1,0 & 0,7 \\
\hline cis9, trans11-CLA & 0,4 & 0,7 & 0,5 & 0,9 & 0,6 & 1,7 & 0,8 \\
\hline
\end{tabular}

1 Pâturage S3 ou S6 = Herbe de prairie naturelle, 3 ou 6 semaines après la mise à l'herbe.

$16: 0,18: 0$ et $18: 1$ cis) augmente les teneurs en 18:0 et 9c-18:1 et diminue fortement les teneurs en AG saturés de 10:0 à 16:0 (Chilliard et al 2001). Toutefois, la plupart de ces suppléments sont maintenant très peu utilisés en Europe, suite à la crise de l'Encéphalopathie Spongiforme Bovine (ESB). La sécrétion de 18:0 dans le lait peut aussi être accrue par l'apport d'AG insaturés à 18 atomes de carbone présents dans les huiles ou les graines végétales en raison de leur hydrogénation partielle, ou totale en 18:0 dans le rumen (Loor et al 2005a). Il en est de même pour le 9c-18:1, en raison soit de sa sécrétion directe (environ 20\%), soit de sa synthèse (environ 80\%) par action de la désaturase mammaire sur le 18:0 (Chilliard et al 2007). Toutefois, ces supplémentations entraînent aussi un accroissement de la production d'AG trans dans le rumen et dans le lait. Les huiles végétales riches en 18:2 n-6 (tournesol, soja) ou 18:3 n-3 (lin) augmentent fortement la teneur en CLA du lait. Cet effet est linéaire lors d'addition de quantités croissantes d'huile à la ration (jusqu'à 3-4\% de la MS, au moins, avec une réponse d'environ 0,4 point par point d'augmentation de la teneur en lipides de la ration) (Chilliard et al 2007, tableau 2).

En Europe, mis à part les fourrages, seule la graine de lin permet des apports importants de 18:3n-3. Les résultats obtenus avec la graine de lin extrudée montrent des augmentations du 18:3 n-3 du lait de 0,3-0,9 g/ $100 \mathrm{~g}$ $\mathrm{AG}$, tout à fait comparables à celles observées avec de l'huile ou de la graine non traitée, mais plus faibles que celles observées chez des vaches pâturant une herbe de bonne qualité (Loor et al 2005a, Akraim et al 2007, Chilliard et al 2007, Ferlay et al 2008). Alors que la supplémentation en huile de lin augmente la teneur du lait en $18: 3 \mathrm{n}-3$, elle diminue ou tend à diminuer les teneurs en EPA et DHA (Loor et al 2005a), confirmant que le 18:3 n-3 n'est pas élongué en EPA et DHA et suggérant qu'il pourrait même limiter leur sécrétion par la glande mammaire.

L'ingestion d'huile de poisson ne modifie pas, ou beaucoup moins nettement que celle d'huiles oléagineuses, les teneurs en $A G$ du lait de 4:0 à 16:0 mais diminue très fortement celles en 18:0 et 9c-18:1 (Chilliard et al 2001). Pour une quantité d'huile similaire distribuée dans la ration, les huiles de poisson augmentent beaucoup plus fortement que les huiles végétales la teneur en 9c, 11t-18:2 du lait (Loor et al 2005b). Ainsi, les teneurs en CLA du lait passent de $0,2-0,6 \%$ avec des régimes témoins à 1,5-2,7\% lors d'une supplémentation par 200-300 g/j d'huile de poisson (Chilliard et al 2001). Les AGPI de ces huiles (EPA, DHA notamment) augmentent en effet la concentration de 11t-18:1 dans le rumen, par inhibition de la réduction de cet $\mathrm{AG}$ en 18:0. Par ailleurs, la sécrétion des AG n-3 à 20 et 22 atomes de carbone (EPA, DPA et DHA) est accrue. L'efficacité $\mathrm{du}$ transfert de la ration au lait est cependant faible (2,6\% pour l'EPA et $4,1 \%$ pour le DHA) en raison de fortes hydrogénations ruminales partielles, en particulier pour l'EPA (Chilliard et al 2001).

Il n'y a que peu de données sur l'influence de l'alimentation sur les différents isomères trans-18:1 et encore moins sur les isomères du 18:2 (conjugués ou non) du lait. La teneur en 9c,11t-18:2 est généralement celle qui varie le plus, en raison de l'importance de sa synthèse mammaire par la $\Delta 9$ désaturase. Toutefois, une ingestion de 18:2 n-6 accroît aussi les 6/7/8t-, 9t-,
$10 \mathrm{t}-18: 1,12 \mathrm{t}-18: 1$ et $10 \mathrm{t} 12 \mathrm{t}-, 9 \mathrm{t} 11 \mathrm{t}-$, 8t10t-, 7t9t-, 10t12c-, 9t11c-, 8t10c-, $7 \mathrm{t} 9 \mathrm{c}-\mathrm{CLA}$, et celle de 18:3 n-3 augmente les 15c-, 13/14t-, 15t-, 16t-18:1, 9c12t-, 9c13t-, 11t15c-18:2, et 9t,11t-, $12 \mathrm{t} 14 \mathrm{t}-, 11 \mathrm{t} 13 \mathrm{t}-, 1214 \mathrm{ct}-, 12 \mathrm{t} 14 \mathrm{c}-$, 11t13c-, 11c13t-CLA (Loor et al 2005a, Roy et al 2006), ainsi que certains isomères conjugués du 18:3 (Akraim et al 2007). Ces effets sur les AG trans sont plus marqués avec les huiles qu'avec les graines et résultent des biohydrogénations ruminales, combinées à la $\Delta 9$ désaturation mammaire des $7 \mathrm{t}-, 12 \mathrm{t}$ - et 13t-18:1 notamment. Par ailleurs, l'huile de poisson accroît les $11 \mathrm{c}-, 6 / 7 / 8 \mathrm{t}$, 9t-, 10t-, 12t-, 13/14t-18:1 et $11 \mathrm{t} 15 \mathrm{c}-$ 18:2 (Loor et al 2005b). Les rôles physiologiques respectifs de ces différents isomères et leur éventuel intérêt nutritionnel pour l'homme n'ont pas ou très peu été étudiés à ce jour.

\section{3 / Interactions entre l'apport de lipides et les autres consti- tuants de la ration}

La supplémentation en huile de lin interagit significativement avec le rapport fourrage/concentré de la ration, augmentant plus fortement les teneurs du lait en 10t-18:1, 11t15c-18:2 et 18:3 n-3 avec un régime riche en aliment concentré, ou augmentant plus les 18:0 et 9c-18:1 et diminuant plus le 16:0 avec un régime riche en foin (Loor et al 2005a). Dans une autre étude sur les huiles de lin et de tournesol (Chilliard et al 2007, tableau 2), le 9c-18:1 du lait augmente plus fortement lorsque la ration est basée sur de l'ensilage d'herbe, comparée à l'ensilage de maïs $(+4,7 v s+2,8 \mathrm{~g} / 100 \mathrm{~g} \mathrm{AG})$ alors que c'est l'inverse pour le 9c11t-CLA $(+0,7 v s+1,3 \mathrm{~g} / 100 \mathrm{~g} \mathrm{AG})$ et les AGPI. La biohydrogénation ruminale semble donc être moins complète avec l'ensilage de maïs, ce qui explique probablement les chutes plus nettes du taux 
Tableau 2. Effet de la nature du fourrage et du supplément lipidique (huile de tournesol, HT, ou de lin, HL, en \% MS ingérée) sur la production de lait, le taux butyreux (TB) et la composition en acides gras (\% AG totaux) du lait de vache (Ferlay et Chilliard non publié, Chilliard et al 2007) (1).

\begin{tabular}{|c|c|c|c|c|c|c|c|c|c|}
\hline Fourrage & Huile $^{(2)}$ & Dose & Lait (kg/j) & TB $(\mathrm{g} / \mathrm{kg})$ & $9 c-18: 1$ & $18: 2 n-6$ & $18: 3 n-3$ & 9c11t-CLA & $18: 3 / 18: 2^{(3)}$ \\
\hline \multirow{5}{*}{$\begin{array}{l}\text { Ensilage } \\
\text { de maïs }\end{array}$} & - & - & 27,9 & 38,9 & 16,0 & 1,7 & 0,3 & 0,6 & 0,17 \\
\hline & HT & 1.5 & 29,2 & 352 & 18.2 & 2,1 & 0.3 & 1.7 & 0.13 \\
\hline & & 3,0 & 30,2 & 31,9 & 20,4 & 2,2 & 0,3 & 2,5 & 0,11 \\
\hline & $\mathrm{HL}$ & 1,5 & 30,2 & 36,9 & 17,5 & 1,7 & 0,5 & 1,4 & 0,29 \\
\hline & & 3,0 & 29,7 & 33,7 & 18,9 & 1,6 & 0,6 & 2,1 & 0,38 \\
\hline \multirow{5}{*}{$\begin{array}{l}\text { Ensilage } \\
\text { d'herbe }\end{array}$} & - & 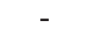 & 25,6 & 37,2 & 17,4 & 1,6 & 0,7 & 0,7 & 0,44 \\
\hline & HT & 1,5 & 25,9 & 36,5 & 21,4 & 1,8 & 0,7 & 1,1 & 0,37 \\
\hline & & 3,0 & 27,7 & 34,6 & 23,4 & 2,0 & 0,6 & 1,9 & 0,31 \\
\hline & $\mathrm{HL}$ & 1,5 & 27,1 & 36,6 & 21,0 & 1,6 & 0,9 & 1,0 & 0,55 \\
\hline & & 3,0 & 26,7 & 35,8 & 22,4 & 1,5 & 0,8 & 1,7 & 0,51 \\
\hline
\end{tabular}

(1) : 20 vaches dans 2 carrés latins $5 \times 5$ répliqués.

(2) : HT riche en 18:2 $n-6, H L$ riche en 18:3 n-3.

(3) : rapport 18:3 n-3/18:2 n-6.

(4) : $47 \%$ ensilage de maïs, $13 \%$ foin de graminées, $40 \%$ concentrés.

(5) : $60 \%$ ensilage d'herbe, $5 \%$ foin de graminées, $35 \%$ concentrés.

butyreux du lait lorsque c'est ce fourrage qui est supplémenté en huile (tableau 2).

Une constante des essais rapportés ci-dessus est l'augmentation de la teneur du lait en 10t-18:1 avec les régimes riches en amidon (concentrés et/ou ensilage de maïs), supplémentés en AGPI. Or ces régimes entraînent une diminution du taux butyreux du lait, liée au moins en partie à l'augmentation de sa teneur en 10t,12c-18:2 (Roy et al 2006). Une conséquence importante de ces modifications est que les synthèses des 11t-18:1 et 9c11t-CLA n'augmentent que faiblement (déviation vers la voie du 10t-18:1) par rapport à ce qui s'observe avec des régimes riches en fibres supplémentés en huiles.

En conclusion, les suppléments d'huiles ou de graines oléagineuses ont des effets similaires, parfois plus marqués, que ceux d'une alimentation au pâturage, mais ils augmentent aussi d'autres isomères trans du 18:1 et du 18:2, notamment lorsqu'ils sont ajoutés à des rations riches en concentrés et/ou en ensilage de maïs. Par ailleurs, les effets à long terme des supplémentations lipidiques (et leurs interactions avec le type de ration utilisé) sur la composition en AG du lait et sur les performances laitières (production laitière, taux butyreux et protéique du lait), la reproduction et la santé des vaches laitières sont encore très mal connus. Une partie des résultats présentés ci-dessus a été confirmée par des études réalisées dans d'autres pays (Dewhurst et al 2006).

\section{4 / La régulation de la sécré- tion lipidique mammaire par les facteurs alimentaires diffère de façon marquée entre la chèvre et la vache}

Nous avons montré que la sécrétion lipidique mammaire, fortement inhibée par des régimes riches en amidon et supplémentés en AGPI chez la vache, ne l'est pas chez la chèvre où elle augmente (Chilliard et al 2003). En effet, chez la vache les teneurs en $11 \mathrm{t}-18: 1$ et $9 \mathrm{c} 11 \mathrm{t}-$ CLA du lait augmentent durant 4 à 6 jours après supplémentation lipidique, puis s'effondrent au profit d'une augmentation très spectaculaire de la teneur en 10t-18:1, ainsi que d'autres isomères trans du C18:1 et du CLA (Roy et al 2006, Chilliard et al 2007). Chez la chèvre, au contraire, les teneurs en 11t-18:1 et 9c11t-CLA du lait augmentent fortement puis restent stables pendant 5 à 10 semaines au moins, alors que la teneur en 10t-18:1 et d'autres isomères trans tels que le t10, c12-18:2 n'augmentent qu'à des niveaux nettement plus faibles que chez la vache. Ces isomères trans sont négativement corrélés à la sécrétion lipidique chez la vache (Roy et al 2006), mais pas ou peu chez la chèvre (Chilliard et al 2006, 2007). Ces résultats suggèrent donc que la chèvre se caractérise par une plus grande stabilité des biohydrogénations ruminales. De plus, une moindre sensibilité aux AG trans de sa lipogenèse mammaire est suggérée par le fait qu'une infusion post-ruminale de t10,c12-18:2 n'inhibe pas la sécrétion lipidique (Andrade et Schmidely 2006), contrairement à la vache.

Ces réponses très différentes entre deux espèces de ruminants a priori très voisines (tableau 3) offrent de nouvelles perspectives de compréhension des mécanismes de régulation du métabolisme ruminal et de la lipogenèse mammaire (Bernard et al 2008), car elles conduisent à remettre en cause ou élargir les paradigmes couramment admis pour la vache. Par ailleurs, la supplémentation en AGPI permet de diminuer comme chez la vache les teneurs du lait de chèvre en AG saturés, et d'accroître les teneurs en $\mathrm{AG}$ potentiellement antiathérogènes ou anticancéreux (oméga 3, CLA), mais les réponses diffèrent pour plusieurs isomères cis ou trans des 18:1 et 18:2 (Schmidely et Sauvant 2001, Chilliard et al 2006, 2007). Les effets potentiels sur la santé des produits caprins et bovins enrichis en ces $\mathrm{AG}$ demandent donc à être évalués de façon spécifique.

\section{2 / Modulation par l'ali- mentation de la composi- tion en acides gras de la viande bovine}

La viande bovine occupe, pour des raisons culturelles notamment, une place importante dans l'alimentation humaine en France (12 kg/habitant/an, Hebel 2007) la situant à la 2 ème place de la consommation relative des viandes $(30 \%)$ après celle de porc $(39 \%)$ et avant celle de volaille $(26 \%)$. Sa production en France, actuellement stable, correspond à 1,6 millions de tonnes équivalent carcasse. Elle provient pour $48 \%$ de vaches (dont $55 \%$ d'origine races à viande), $31 \%$ de jeunes bovins, $13 \%$ de génisses et $8 \%$ de boeufs (données Office de l'Elevage 2006). 
Tableau 3. Réponses respectives de la vache et de la chèvre laitière à des régimes riches en amidon et en acides gras polyinsaturés (Chilliard et al 2003, 2007, Andrade et Schmidely 2006, Bernard et al 2008).

\begin{tabular}{|l|c|c|}
\hline & Vache & Chèvre \\
\hline Chute de taux butyreux du lait et de sécrétion lipidique mammaire & Oui & Non \\
Stabilité de réponse des acides vaccénique (11t-18:1) et ruménique (9c11t-CLA) & Non & Oui \\
Production de 10t-18:1 & Forte & Faible \\
Inhibition de la lipogenèse mammaire par le 10t12c-CLA & Oui & Non \\
Réponse de l'acide oléique (9c-18:1) & Forte & Moyenne \\
Rapport 9c-18:1/18:0 du lait & Elevée & Faible \\
Réponse de l'acide linolénique (18:3 n-3) à un apport de graine de lin & Moyenne & Elevée \\
\hline
\end{tabular}

Les premières études sur les lipides de la viande bovine et leurs AG réalisées à l'INRA ont surtout porté dans les années 80-90 sur les modifications de leur structure chimique (lipolyse, peroxydation des AG, interactions avec les réactions de Maillard) par les procédés technologiques en relation avec les conditions de cuisson et les conséquences sur la flaveur via la production d'arômes (Gandemer 1999). Au cours de cette même période, les recherches de l'INRA sur la modulation de la composition en $A G$ de la viande bovine par des facteurs d'élevage ont été initiées chez le veau de boucherie recevant des aliments d'allaitement très riches en matières grasses puis, à partir de 1998, chez le bovin à viande adulte en finition en fonction du type de ration de base et de l'origine et du mode d'apport de suppléments lipidiques (Geay et al 2001). Cela s'explique par la nécessité d'une meilleure maîtrise de la composition et de la teneur en AG des différentes viandes et abats face à une image nutritionnelle chroniquement négative auprès du corps médical et des consommateurs, de plus en plus soucieux des effets des aliments sur la santé humaine.

\section{1 / Veau de boucherie}

Dès le début des années 50, les lipides de l'aliment d'allaitement ont été constitués (pour l'essentiel) de graisses animales (suif, saindoux) bien équilibrées pour répondre aux besoins de croissance du veau préruminant (Toullec et al 2003). Suite à la crise de la vache folle à la fin des années 90, les graisses animales ont, en majeure partie, été remplacées par des matières grasses d'origine végétale. Les conditions d'emploi de ces huiles ont été analysées tant pour leur impact sur l'efficacité alimentaire et les performances de croissance que pour leurs effets sur la composition en AG des viandes et gras de dépôt.

Ainsi, chez le veau dès l'âge de 3 semaines, l'emploi d'huile de soja comme seule source d'AG a fortement augmenté la teneur en 18:2 n-6 des phospholipides ( $\times 2$ ) et surtout des triglycérides (X 16) du muscle Longissimus thoracis (LT, faux filet) au détriment du 18:1 n-9. Cependant, l'emploi de cette huile, très riche en AGPI de la famille n-6, entraîne l'apparition de refus consécutifs à l'installation d'une infiltration lipidique du foie (stéatose) interdisant l'emploi, à l'état natif, d'huiles très riches en AGPI (Bauchart et al 1996). De même, l'emploi exclusif d'huile de coprah, riche en AG saturés de type 12:0 et 14:0 (source d'énergie pour la croissance du veau) entraîne les mêmes anomalies métaboliques que l'huile de soja. De plus, elle favorise le dépôt de ces AG saturés pro-athérogènes au détriment $\mathrm{du}$ 18:1 n-9 conduisant à une dépréciation de la qualité diététique de la viande (Bauchart et al 1999). La nécessité d'un apport modéré d'AGPI dans l'aliment d'allaitement pour veau conduit actuellement à l'emploi d'huiles végétales partiellement hydrogénées, mais entraîne le dépôt dans la viande de certains isomères d'AG monoinsaturés trans (formes $\Delta 9$, 10 et 12trans) considérés comme proathérogènes pour l'homme (Bauchart et Bispo-Villar, données non publiées). L'emploi de margarines dénuées d'AG monoinsaturés trans, récemment produites par l'industrie, pourrait éviter la détérioration de la valeur diététique des lipides déposés dans la viande chez le veau de boucherie.

\section{2 / Bovin ruminant en finition}

\section{a) Effets d'une ration à base d'herbe}

La viande produite à partir de bovins élevés à l'herbe dans les zones traditionnelles d'élevage bénéficie d'une image de qualité favorable auprès du consommateur. Les travaux de l'INRA montrent que, par rapport à un régime à base d'ensilage de maïs, un régime à base d'herbe ne modifie pas la teneur en lipides des muscles Rectus abdominis (RA, bavette de flanchet) et Semitendinosus (ST, rond de gite). En revanche, il favorise l'enrichissement en AGPI n-3 (RA: 2,7 vs 0,9\%; ST: 6,1 vs $1,9 \%$ respectivement) de ces muscles au détriment du 18:1 n-9 contribuant, notamment dans le muscle $\mathrm{ST}$, à élever les teneurs en 18:3n-3 (x 4), le 20:5 n-3 (x 4) et 22:5 n-3 (x 2) et ainsi diminuer le rapport AGPI n-6/AGPI n-3 $(1,9$ vs 7,0 et 1,4 vs 5,6 respectivement) (Bauchart et al 2001). L'ensemble de ces modifications pourrait contribuer à l'amélioration de la valeur santé des $A G$ de la viande bovine pour l'homme, notamment dans le domaine de la prévention de l'athérosclérose.

\section{b) Effets du rapport fourrage/aliment} concentré de la ration

La nature et la proportion de fourrages apportés en complément de l'aliment concentré modifient également la composition en AG de la viande. Ainsi, chez le taurillon en finition, une ration à base de paille et d'aliment concentré (30/70) comparée à une ration ensilage de maïs/aliment concentré (60/40) pourrait améliorer la valeur nutritionnelle du muscle Rectus abdominis i) en diminuant la teneur en $\mathrm{AG}$ saturés (-13\%), notamment le 16:0 pro-athérogène $(-14 \%)$ et en accroissant les AGPI $\mathrm{n}-6(+52 \%)$ et $\mathrm{n}-3(+57 \%), i i)$ en favorisant le dépôt de CLA 9cis,11trans $(+57 \%)$ et de son précurseur 18:1 11 trans $(+48 \%)$ considérés comme potentiellement favorables à la santé de l'Homme (prévention des cancers, MCV..., Bauchart et al 2005).

\section{c) Effets des suppléments lipidiques}

Initialement introduits dans la ration en tant que sources de composés de haute densité énergétique permettant d'engraisser les animaux et d'acquérir une conformation de carcasse satisfaisante avant l'abattage, les suppléments lipidiques présentent l'intérêt supplémentaire de pouvoir améliorer la valeur nutritionnelle des AG des viandes pour le consommateur.

Ainsi, dans le cadre du programme européen HealthyBeef du 5 ème PCRD (2000-2003), des essais d'alimentation sur bouvillons pendant $70 \mathrm{j}$ avec une ration à base de foin et d'aliment concentré (45/55) supplémentée en 
lipides (4\% MS) par des graines de lin extrudées (riches en 18:3n-3) ont mis en évidence une amélioration de la valeur nutritionnelle de la viande (bavette de flanchet) traduite par une diminution du rapport n-6/n-3 des AGPI dans les lipides neutres (- 18\%) et surtout polaires (-38\%) et une augmentation dans les lipides neutres des teneurs en 18:1 11trans $(+42 \%)$ et en CLA (+ 50\%), (Durand et al 2005). Ces résultats sont en accord avec les données de Scollan et al (2005) et de Wood et al (2007) chez le bovin à l'engraissement recevant des rations supplémentées en lipides riches en AGPI n-3 (rations à base d'herbe, ou supplémentées en huiles de lin ou de poisson).

L'impact de l'apport de suppléments lipidiques par la graine de lin varie selon la nature de la ration de base (Bauchart et al 2005). Ainsi, une supplémentation en graines de lin extrudées a une effet positif plus marqué sur les teneurs en CLA et en 18:1 11trans de la viande quand elle est réalisée sur une ration paille-concentré $(30 / 70)$ que sur une ration ensilage de maïs-concentré (60/40), mais les deux régimes conduisent à des effets comparables sur le 18:3 n-3. L'introduction dans ces régimes d'un haut niveau de vitamine $\mathrm{E}$ (2500 unités/animal/j) pour assurer une meilleure protection des AGPI vis-à-vis de la peroxydation entraîne des modifications de la composition en AG de la viande. Ainsi, la baisse des teneurs en AGPI de type 18:2 n-6 (- 20\%) et 18:3 n-3 (- 6 à - 13\%) observée après apport de vitamine $\mathrm{E}$ pourrait s'expliquer par une stimulation de la biohydrogénation ruminale favorisant la production de 18:1 11trans dont le dépôt a été accru dans la viande $(+17 \%$ à $36 \%$; De La Torre et al 2006). L'emploi de graines de colza, source de 18:2 n-6 (22\%) et 18:3 n-3 (11\%), favorise le dépôt de ces $\mathrm{AG}$ dans la viande chez le taurillon sans changer notablement le rapport n6/n-3 ni les teneurs en CLA et en 18:1 11trans (Bauchart et al 2005).

La potentialité de captage des AGPI n-3 par les muscles a été estimée par l'administration en continu (par voie duodénale pendant $70 \mathrm{j}$ ) chez le bouvillon d'une quantité d'huile de lin $(400 \mathrm{~g} / \mathrm{j})$ équivalente à celle consommée sous forme de graines extrudées. Les résultats montrent une très forte augmentation de la teneur en 18:3 n-3 par rapport au traitement témoin sans ajout de lipides $(8,0 \%$ vs $0,5 \%$ des $A G$ totaux) (Durand et al 2005). Cependant, un tel traitement conduit à l'ap- parition d'un goût de poisson très prononcé de la viande totalement indésirable pour le consommateur, ce qui souligne la nécessité d'une bonne maîtrise du niveau d'incorporation des AGPI n-3 dans la viande.

\section{3 / Modulation par l'ali- mentation de la composi- tion en acides gras de la viande de porc}

Les études sur la qualité nutritionnelle de la viande de porc sont récentes. Une priorité a en effet longtemps été donnée à la qualité technologique de la matière première en raison de l'importance de la transformation qui est de près de $70 \%$ chez le porc. Un des objectifs initiaux des études sur la qualité nutritionnelle entreprise dans les années 90 a été d'apporter des informations sur la teneur réelle en lipides et la composition en AG de la viande. En effet, la viande de porc continuait de véhiculer une image de viande grasse alors que la masse adipeuse globale de la carcasse avait été déjà fortement réduite : elle est passée d'environ $40 \%$ du poids de la carcasse à près de $20 \%$ entre les années 60 et 2000. De plus, la teneur en lipides des tissus adipeux a été également réduite de 10 à $15 \%$ selon la localisation des tissus.

Un objectif récent, basé sur la relation qui existe entre la nature des $\mathrm{AG}$ de l'aliment et ceux déposés dans la viande de porc, a été d'introduire dans l'alimentation du porc des AG jugés favorables pour la santé humaine afin de les apporter dans l'assiette du consommateur via la viande et les produits transformés. Deux grandes familles d'AG ont paru intéressantes. L'une concerne le CLA (Acide Linoléique Conjugué). L'autre les AG $\mathrm{n}-3$, en particulier l'acide $\alpha$ linolénique (18:3n-3).

\section{1 / Les acides gras cibles pour l'alimentation humaine}

\section{a) L'acide linoléique conjugué}

A coté de leurs effets potentiellement positifs sur la santé humaine, certains isomères du CLA permettraient également d'induire une diminution de la masse adipeuse en réduisant la synthèse des lipides chez le porc (Corino et al 2006) ; toutefois l'isomère t10,c12 largement présent dans les mélanges commerciaux pourrait aussi avoir des effets négatifs (résistance à l'insuline...) montrés chez les rongeurs (Wahle et al 2004). Ces CLA apportés dans l'alimentation du porc sont retrouvés dans la viande. Avec un apport de 0,5\% de CLA dans l'aliment, la teneur en lipides du tissu est diminuée de 8 à $10 \%$, et le dépôt de CLA est voisin de $430 \mathrm{mg}$ pour $100 \mathrm{~g}$ de tissu adipeux (Corino $\mathrm{et}$ al 2006).

\section{b) L'acide $\alpha$ linolénique (ALA)}

Les travaux conduits sur l'ALA depuis 2002 ont prouvé la faisabilité de l'augmentation de la teneur des produits porcins en cet $\mathrm{AG}$, et ont montré que la transformation de la viande de porc n'altérait pas les AGPI n-3 (Enser et al 2002, Mourot 2004, Corino et al 2008, Guillevic et al 2007). Le lin et le colza ont été utilisés, mais d'autres sources sont également recherchées, comme le chanvre, dont l'huile donne des résultats intéressants. La teneur en AGPI n-3 retrouvée dans la viande est supérieure à celle obtenue chez des porcs recevant de l'huile de colza (250 à $290 \mathrm{mg}$ C18:3 n-3/100 g de côte de porc, vs 150 à $190 \mathrm{mg}$, Mourot et al 2008). Toutefois la teneur est moins importante que chez des porcs recevant du lin (dépôt de 500 à $600 \mathrm{mg}$ $18: 3 \mathrm{n}-3$ par $100 \mathrm{~g}$ de côte de porc) du fait que l'huile de chanvre contient moins de 18:3 n-3 que celle de lin. La graine de chanvre peut donc être une autre source non négligeable d'apport de 18:3 n-3 dans l'alimentation animale.

Il existe des relations très fortes ou assez fortes entre la teneur en ALA de l'aliment et les dépôts de ALA, EPA, DPA par gramme de lipides de la viande (figure 1). En revanche, il existe peu d'effet pour le DHA ce qui montre la faible capacité tissulaire de conversion du DPA en DHA, comme chez la plupart des animaux, y compris le poisson.

\section{2 / Conséquences des modifi- cations de l'alimentation du porc sur les lipides ingérés par l'Homme}

Les résultats précédents montrent que l'incorporation de certains $\mathrm{AG}$ dans l'alimentation du porc permet de les retrouver en partie dans la viande et les produits transformés. Ces pratiques sont donc possibles, mais ont-elles toutes le même intérêt ? 
Figure 1. Relation entre la quantité d'ALA ingéré ( $g / j)$ et les teneurs de la viande de porc en AG $n-3$ ( $A L A, E P A, D P A, D H A)$. Chaque point représente la moyenne d'un lot de 10 à 16 porcs élevés dans des conditions identiques (Mourot et al données non publiées).

ALA

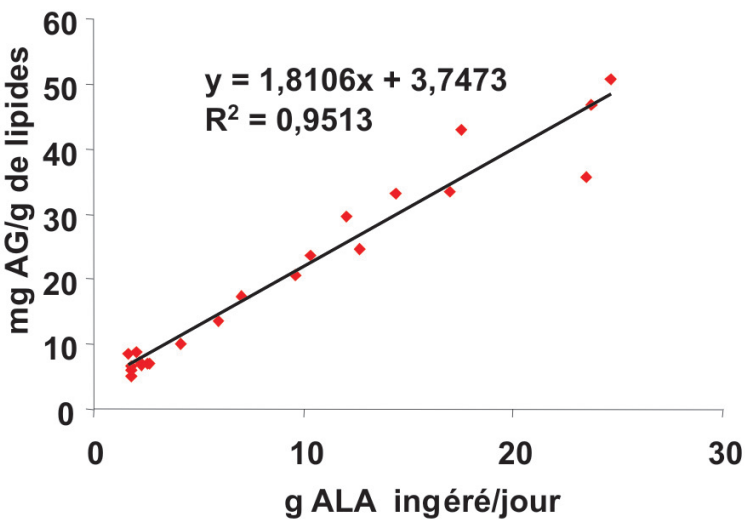

DPA

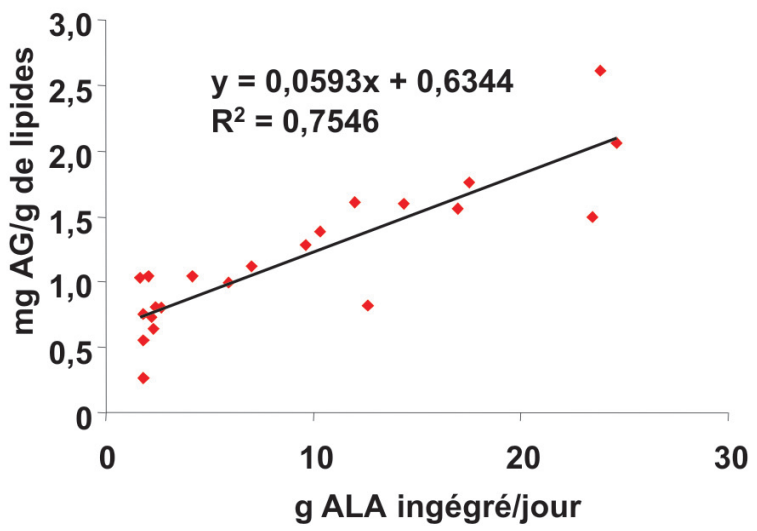

EPA

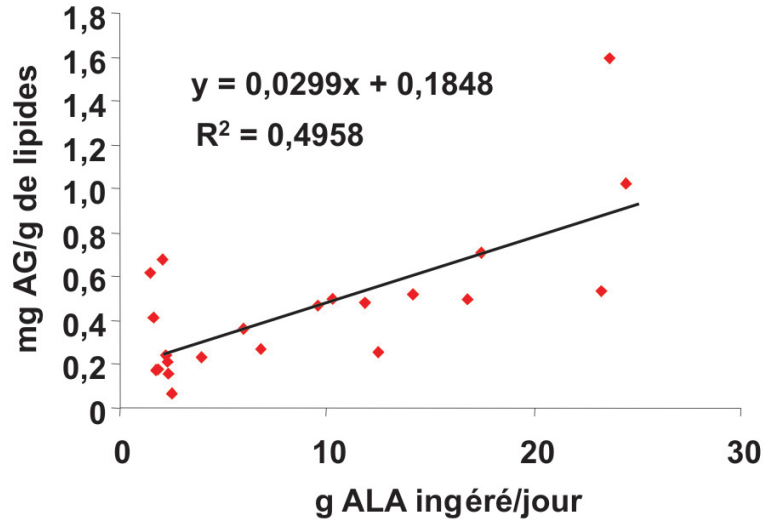

DHA

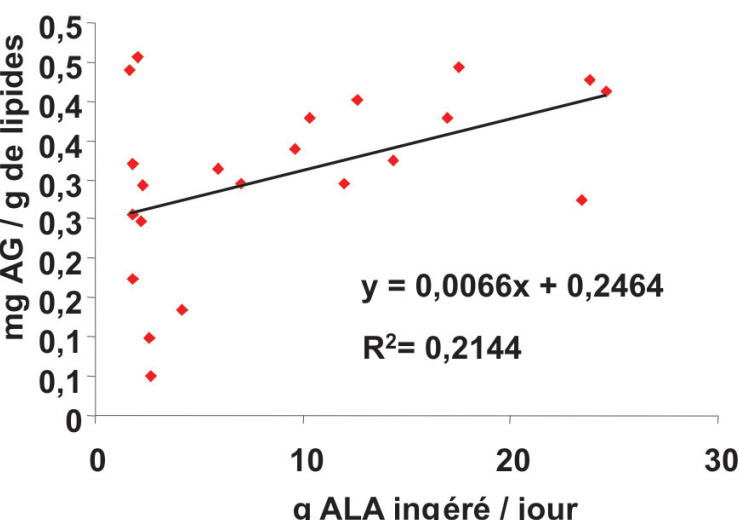

Pour les CLA, il semble que cela ne soit pas le cas. Contrairement au ruminant, le porc n'est pas capable de les synthétiser. Ils doivent donc être apportés par l'alimentation. Or, les CLA produits par synthèse sont apportés sous forme de mélange et certains CLA ne semblent pas avoir les effets biologiques positifs escomptés (Wahle et al 2004). De plus le coût est très élevé. Cette voie de supplémentation, un moment envisagée chez le porc, est actuellement abandonnée.

En revanche, la supplémentation en AGPI n-3 de l'aliment du porc est en plein développement (Wood et al 2007). L'intérêt est validé par des études réalisées chez l'homme montrant une diminution du poids corporel et une baisse de la triglycéridémie après consommation des produits carnés enrichis naturellement en AGPI n-3 via l'alimentation animale (Weill et al 2002). Les estimations faites sur la comparaison de produits standards vs produits enrichis montrent un accroissement de la teneur en n-3 entre 4 et
7 fois, selon les produits (Guillevic et al 2007). $100 \mathrm{~g}$ de côte de porc peuvent ainsi apporter près de $400 \mathrm{mg}$ d'ALA.

En conclusion, il est possible par l'alimentation du porc de modifier la composition en $A G$ de sa viande pour avoir un produit potentiellement meilleur pour la santé de l'homme. Les recherches sont encore à poursuivre en particulier pour les périodes et durées de distribution des régimes enrichis et pour une protection accrue des AGPI n-3 dans la viande et produits transformés en recherchant les antioxydants les plus efficaces et en essayant d'améliorer la biodisponibilité en jouant sur la compétition n-6/n-3.

Une rillette aux oméga 3 n'est donc pas une aberration. Elle permettrait de faire consommer à l'homme davantage d'AGPI n-3 sans qu'il ne change ses habitudes alimentaires. Toutefois, une réduction de la teneur en lipides des produits ne doit pas être négligée pour obtenir un meilleur équilibre des rations.

\section{4 / Modulation par l'ali- mentation de la composi- tion en acides gras de la viande de lapin}

Des approches similaires à celles développées sur le porc ont été conduites à la Station de Recherches Cunicoles de Toulouse et à l'UMR SENAH. Comme pour le porc, il est possible d'introduire des CLA dans l'aliment du lapin et les retrouver ensuite dans l'assiette du consommateur (Corino et al 2002). Mais cette voie ne sera vraisemblablement pas exploitée, pour des raisons identiques à celles du porc.

Pour les AGPI n-3, la voie de l'apport alimentaire semble par contre promet- 
teuse (Mourot et al 2006, Combes et Cauquil 2006) ; des produits sont déjà disponibles et les recherches se poursuivent au niveau industriel. L'apport de luzerne dans l'alimentation du lapin, combinée avec une supplémentation en graines de lin permet de multiplier par 3 à 5 les teneurs en AGPI n-3 de la viande. Toutefois cette dernière aura moins d'incidence sur la modification de la consommation des lipides chez l'Homme que celle du porc en raison de sa faible consommation $(2,2 \mathrm{~kg} / \mathrm{an} / \mathrm{per}-$ sonne) et d'une teneur en lipides plus faible. Mais cette viande peut venir en complément des autres sources alimentaires d'AGPI n-3.

\section{5 / Modulation par l'ali- mentation de la composi- tion en acides gras de la viande de volailles}

Les oiseaux sont avant tout des granivores et leur besoin énergétique est en grande partie couvert par les glucides alimentaires. C'est le foie qui assure la synthèse endogène d'AG à partir des glucides ingérés, dont la dégra-dation aboutit à la formation d'acétyl CoA puis à la synthèse de palmitate (16:0). Celui-ci est ensuite élongué puis désaturé par une $\Delta 9$ désaturase pour aboutir à l'acide oléique (18:1 n-9). Les oiseaux, incapables de transformer l'acide palmitique en acides linoléique et linolénique en l'absence des désaturases correspondantes, sont tributaires des apports alimentaires en ces AG. Chez le poulet, la nature des AG déposés dans les tissus dépend donc des apports en substrats alimentaires : glucides ou lipides.

Les travaux réalisés sur l'œuf (dont nous consommons environ $3 \mathrm{~g}$ d'AG par jour) ont montré que ce produit

Figure 2. Cinétique d'incorporation du 18:3 n-3 dans les triglycérides du Pectoralis major de poulet en fonction de la teneur en 18:3n-3 des lipides alimentaires et de l'âge.

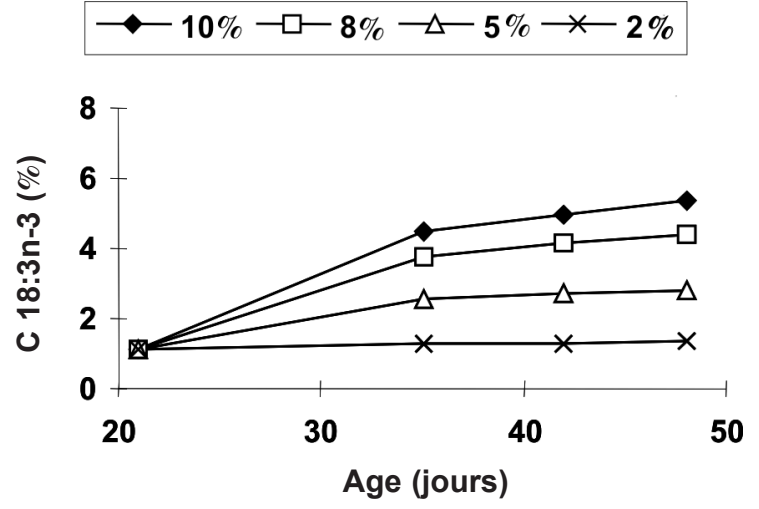

reflète assez fidèlement la nature des lipides ingérés par la pondeuse (Van Elswyk 1997). Aucun travail n'a été entrepris à l'INRA pour étudier l'intérêt des CLA chez les oiseaux. A l'inverse, chez le poulet, plusieurs essais ont été réalisés par l'URA à Nouzilly et le LEIMA à Nantes dans les années 90-2000 (Gandemer et al 1999, Viau et al 1999, Lessire 2001). Ils avaient pour objectifs, d'une part de mesurer la cinétique d'incorporation des acides linoléique et linolénique alimentaires dans les tissus adipeux et musculaires et d'autre part d'établir les relations entre les AG ingérés et ceux déposés dans les compartiments corporels. Au total, 35 aliments isolipidiques (8\%) ont été constitués en utilisant des proportions variables de différents corps gras : suif, huiles de palme, coprah, tournesol, tournesol oléique et lin, pour faire varier les apports en $18: 2$ n-6 (de 20 à $60 \%$ des lipides de l'aliment) à teneur en 18:3 n-3 constante $(2 \%)$ et ceux en 18:3 n-3 (de 2 à $10 \%$ des lipides) pour une teneur en $18: 2 n-6$ de $40 \%$.

Un minimum de 15 jours de distribution des aliments est nécessaire pour obtenir le taux maximum d'incorporation de l'AG testé dans les triglycérides $\mathrm{du}$ filet de poulet (Pectoralis major), ce taux maximum étant proportionnel à

Pour ce qui concerne la composition finale des tissus à l'abattage, les modifications engendrées par l'aliment sont importantes au niveau du gras abdominal et des triglycérides du muscle (cuisse et filet) : les relations sont linéaires et les coefficients de corrélations sont élevés $(0,92-0,98)$. Les augmentations des teneurs des tissus en 18:2 n-6 et 18:3 n-3, liées aux apports alimentaires en ces AG, sont majoritairement contrebalancées par une diminution de la teneur en C18:1 $(r=-0,99)$. l'apport alimentaire (figure 2).

ations sont d'ampleur moindre, mais restent proportionnelles aux apports alimentaires, s'exerçant principalement au détriment de l'acide oléique et de l'autre famille d'AGPI. Ainsi une alimentation enrichie en $\mathrm{AG}$ de la série n-3 appauvrit les tissus en AG n-6 et inversement.

La nature des lipides ingérés a aussi un effet sur la qualité de la carcasse des poulets. Dans les essais précédents, la note de présentation $(1=$ gras huileux et mou à $5=$ gras ferme et $\mathrm{sec}$ ) a ainsi été bien corrélée à la teneur en 16:0 $(\mathrm{r}=0,78)$ et en AGPI majeurs $(\mathrm{r}=-0,78)$. Pour les abattoirs, une présentation de carcasse convenable correspond à une note au moins égale à 3 qu'il est possible d'obtenir si les apports en 18:1+18:2 n-6+18:3 n-3 ne dépassent pas $72 \%$ des AG alimentaires.

En définitive, ces travaux ont non seulement mis en relation la quantité et la nature des lipides ingérés avec la composition en AG de la carcasse de poulet (en accord avec les résultats obtenus dans d'autre pays, Rymer et Givens 2005), mais ont aussi permis aux abattoirs et fabricants d'aliment d'anticiper la suppression des produits d'origine animale (graisses et farines) riches en AG saturés.

\section{Conclusion}

L'alimentation permet de faire varier largement, et de façons diverses, la composition en AG des produits animaux. Les progrès récents des connaissances sur les mécanismes de synthèse des différents AG (digestion et métabolisme), et sur leurs effets physiologiques potentiels chez l'Homme, stimulent fortement les recherches en cours et leurs applications potentielles.

Il n'existe toutefois que peu d'études mesurant finement la composition en AG des produits, notamment chez les ruminants qui présentent une grande complexité de réponse des AG dérivés des biohydrogénations ruminales, et nécessitent de ce fait un développement particulier des méthodes analytiques. Des études comparant systématiquement différents fourrages, concentrés, suppléments lipidiques (huiles, graines, traitements technologiques) et leurs interactions restent à conduire chez les espèces monogastriques et chez les ruminants. Il est, de ce fait, encore difficile d'établir précisément les lois de réponse à l'alimentation de l'ensemble 
des AG d'intérêt potentiel pour l'Homme. De plus, les effets potentiels d'un grand nombre de ces AG sont encore inconnus chez le rongeur et l'Homme.

Chez les monogastriques, les importantes possibilités de modulation de la composition des produits grâce aux pratiques d'élevage et d'alimentation devraient inciter davantage à développer des travaux en particulier chez les animaux producteurs de viande ou d'œufs élevés sur des parcours extérieurs (labels, bio). L'impact de ces par- cours et du couvert végétal consommé sur la composition de ces produits est en effet très mal connu. Ces travaux pourraient en outre apporter la possibilité de tracer leur origine, comme cela a été montré récemment pour le lait de vache, en utilisant sa composition fine en AG (Engel et al 2007).

Dans la mesure où les recommandations nutritionnelles pour l'homme peuvent encore varier dans les prochaines années, les nutritionnistes des animaux de rente doivent continuer à étudier les lois de réponses d'un grand nombre d'AG majeurs et mineurs, et modéliser leurs voies de synthèse. En outre, les effets secondaires potentiels des différentes pratiques alimentaires sur la santé et la reproduction des animaux, et sur la qualité technologique, sensorielle et sanitaire (transfert éventuel de facteurs antinutritionnels présents dans certaines graines, variations de nutriments à effet pro-oxydant, apports alimentaires d'antioxydants, etc), et l'image de leurs produits, demandent à être mieux évalués.

\section{Références}

AFSSA, 2005. Risques et bénéfices pour la santé des AG trans apportés par les aliments. Recommandations. http://www.afssa.fr.

Akraim F., Nicot M.C., Juaneda P., Enjalbert F., 2007. Conjugated Linolenic Acid (CLnA), conjugated linoleic acid (CLA) and other biohydrogenation intermediates in plasma and milk fat of cows fed raw or extruded linseed. Animal, 1, 835-843.

ANC, 2001. Apports Nutritionnels Conseillés pour la population française. AFSSA (Ed), Tec et Doc, Paris, France.

Andrade P.V.D., Schmidely P., 2006. Effect of duodenal infusion of trans 10 , cis 12-CLA on milk performance and milk fatty acid profile in dairy goats fed high or low concentrate diet in combination with rolled canola seed. Reprod. Nutr. Dev., 46, 31-48.

Bauchart D., Ortigues I., Hocquette J.F., Gruffat D., Durand D., 1996. Energy and fat metabolism of the liver, the digestive tract and muscles of the veal calf: transport, processing, energy consumption, fixation by tissues. In: Veal, perspectives to the year 2000, Proc. Int. Symp., French Calf Fed. (Ed), 12 -13 septembre, Le Mans, France, 255-290.

Bauchart D., Durand D., Gruffat-Mouty D., Piot C., Graulet B., Chilliard Y., Hocquette J.F., 1999. Transport sanguin et métabolisme tissulaire des lipides chez le veau de boucherie ; effets du remplacement du suif par de l'huile de coprah dans l'aliment d'allaitement. INRA Prod. Anim., $12,273-285$.

Bauchart D., Durand D., Gruffat D., Dozias D., Ortigues-Marty I., Micol D., 2001. Effets d'un régime à base d'herbe sur la teneur et la composition en acides gras des lipides des muscles et du foie chez le bouvillon à l'engrais. Renc. Rech. Rum., 8, 108.

Bauchart D., Gladine C., Gruffat D., Leloutre L., Durand D., 2005. Effects of diets supplemented with oil seeds and vitamin $\mathrm{E}$ on specific fatty acids of Rectus abdominis muscle in charolais fattening bulls. In: Indicators of milk and beef quality, EAAP, Academic Publishers (Ed), Wageningen, Netherlands, 112, 431-436.

Bernard L., Leroux C., Chilliard Y., 2008. Expression and nutritional regulation of lipogenic genes in the ruminant lactating mammary gland. In: Bioactive components of milk. Bösze Z. (Ed), Springer, USA. Adv. Exp. Med. Biol., 606, 67-108.

Bourre J.M., Pascal G., Durand G. 1984. Alterations in the fatty acid composition of rat brain cells (neurons, astrocytes and oligodendrocytes) and of subcellular fractions (myelin and synaptosomes) induced by a diet devoid of n-3 fatty acids. J. Neurochem., 43, 342-348.

Chilliard Y., 1993. Dietary fat and adipose tissue metabolism in ruminants, pigs, and rodents: a review. J. Dairy Sci., 76, 3897-3931.

Chilliard Y., Gagliostro G., Flechet J., Lefaivre J., Sebastian I., 1991. Duodenal rapeseed oil infusion in early and midlactation cows. 5. Milk fatty acids and adipose tissue lipogenic activities. J. Dairy Sci., 74, 1844-1854.

Chilliard Y., Doreau M., Gagliostro G., Elmeddah Y., 1993. Protected (encapsulated or calcium soaps) lipids in dairy cow diets. Effects on production and milk composition. INRA Prod. Anim., 6, 139-150.

Chilliard Y., Ferlay A., Doreau M., 2001. Effect of different types of forages, animal fat or marine oils in cow's diet on milk fat secretion and composition, especially conjugated linoleic acid (CLA) and polyunsaturated fatty acids. Livest. Prod. Sci., 70, 31-48.

Chilliard Y., Ferlay A., Rouel J., Lamberet G. 2003. A review of nutritional and physiological factors affecting goat milk lipid synthesis and lipolysis. J. Dairy Sci., 86, 1751-1770.

Chilliard Y., Rouel J., Ferlay A., Bernard L., Gaborit P., Raynal-Ljutovac K., Lauret A., Leroux C., 2006. Optimising goat's milk and cheese fatty acid composition. In: Improving the fat content of foods, Woodhead Publ Ltd (Ed), UK, Chapter 12, 281-312.

Chilliard Y., Glasser F., Ferlay A., Bernard L., Rouel J., Doreau M., 2007. Diet, rumen biohydrogenation, cow and goat milk fat nutritional quality: a review. Eur. J. Lipid Sci. Tech., 109, 828-855.

Combes S., Cauquil L., 2006. Viande de lapin et oméga 3 : Une alimentation riche en luzerne permet d'enrichir la viande des lapins en oméga 3. Viandes et Produits Carnés, 25, 31-35.

Corino C., Mourot J., Magni S., Pastorelli G., Rosi F., 2002. Influence of dietary conjugated linoleic acid (CLA) on growth, meat quality, lipogenesis, and plasma leptin and some physiological variables of lipidic metabolism in rabbits. J. Anim. Sci., 80, 1020-1028.

Corino C., Pastorelli G., Douard V., Rossi R., Musella M., Mourot J., 2006. L'acide linoléique conjugué (ALC ou CLA) en nutrition porcine. INRA Prod. Anim., 19, 39-46.
Corino C., Musella M., Mourot J., 2008. Influences of extruded linseed on growth, carcass composition and meat quality of pigs slaughtered at 110 and $160 \mathrm{~kg}$ liveweight. J. Anim. Sci., 81, 2219-2229.

Couvreur S., Hurtaud C., Marnet P.G., Faverdin P., Peyraud J.L., 2007. Composition of milk fat from cows selected for milk fat globule size and offered either fresh pasture or a corn silage-based diet. J. Dairy Sci., 90, 392-403.

Darnton-Hill I., Nishida C., James W.P., 2004. A life course approach to diet, nutrition and the prevention of chronic diseases. Public Health Nutr., 7, 101-121.

De La Torre A., Debiton E., Durand D., Chardigny J.M., Berdeaux O., Loreau O., Barthomeuf C., Bauchart D., Gruffat D., 2005. Conjugated linoleic acid isomers and their conjugated derivatives inhibit growth of human cancer cell lines. Anticancer Res., 25, 3943-3949.

De La Torre A., Gruffat D., Durand D., Micol D., Peyron A., Scislowski V., Bauchart D., 2006. Factors influencing proportion and composition of CLA in beef. Meat Sci., 73, 258-268.

Dewhurst R. J., Shingfield K. J., Lee M. R. F. Scollan, N. D., 2006. Increasing the concentrations of beneficial polyunsaturated fatty acids in milk produced by dairy cows in high-forage systems. Anim. Fd Sci. Technol., 131, 168-206.

Durand G., Pascal G., Gounelle de Pontanel H., 1979. Traitement de l'hypercholestérolémie chez le rat mâle par introduction dans la ration d'huile de soja supplémentée ou non par de l'huile de sardine. Ann. Nutr. Alim., 33, $687-$ 706.

Durand D., Scislowski V., Chilliard Y., Gruffat D., Bauchart D., 2005. High fat rations and lipid peroxidation in ruminants; consequences on animal health and quality of products. In: Indicators of milk and beef quality, EAAP, Academic Publishers (Ed), Wageningen, Netherlands, 112, 137-150.

Engel E., Ferlay A., Cornu A., Chilliard Y., Agabriel C., Bielicki G., Martin B., 2007. Relevance of isotopic and molecular biomarkers for the authentication of milk according to production zone and type of feeding of the cow. J. Agric. Fd Chem., 55, 9099-9108.

Enser M., Richardson R.I., Wood J.D., Gill B.P., Sheard P.R., 2002. Feeding linseed to increase the n-3 PUFA of pork: fatty acid composition of muscle, adipose tissue, liver and sausages. Meat Sci., 55, 201-212. 
Ferlay A., Martin B., Pradel P., Coulon J.B. Chilliard Y., 2006. Influence of grass-based diets on milk fatty acid composition and milk lipolytic system in Tarentaise and Montbeliarde cow breeds. J. Dairy Sci., 89, 4026-4041.

Ferlay A., Agabriel C., Sibra C., Journal C., Martin B., Chilliard Y., 2008. Tanker milk variability of fatty acids according to farm feeding and husbandry practices in French semi-mountain area. Dairy Sci. Technol., 88, sous presse.

Gandemer G., 1999. Lipids and meat quality: lipolysis, oxidation, Maillard reaction and flavour. Sci. Alim., 19, 439-458.

Gandemer G., Viau M., Maillard N., Lessire M., Juin H., 1999. Lipides alimentaires et qualité de la viande de poulet : influence de l'apport de quantité croissante d'acide linolénique (18:3 n-3). 3 èmes Journ. Rech. Avicole, 23-25 Mars, Saint-Malo, France, 403-406.

Geay Y., Bauchart D., Hocquette J.F., Culioli J., 2001. Effects of nutritional factors on biochemical, structural and metabolic characteristics of muscles in ruminants; consequences on dietetic value and sensorial qualities of meat. Reprod. Nutr. Dev., 41, 1-26.

Guillevic M., Le Minous A.E., Blochet J.E., Damon M., Mourot J., 2007 Effet de rations enrichies en acides gras oméga 3 ou oméga $6 \mathrm{chez}$ le porc : impacts sur la qualité nutritionnelle et la qualité sensorielle des produits transformés. Journ. Rech. Porcine Fr., 39, 223-230.

Hebel P., 2007. Comportements et consommations alimentaires en France. Lavoisier (Ed), 144p.

Jensen R.G., 2002. The composition of bovine milk lipids: January 1995 to December 2000. J. Dairy Sci., 85, 295-350.

Lessire M., 2001. Matières grasses alimentaires et composition lipidique des volailles. INRA Prod. Anim., 14, 365-370.

Loor J.J., Ferlay A., Ollier A., Doreau M., Chilliard Y., 2005a. Relationship among trans and conjugated fatty acids and bovine milk fat yield due to dietary concentrate and linseed oil. J. Dairy Sci., 88, 726-740.

Loor J.J., Doreau M., Chardigny J.M., Ollier A., Sebedio J.L., Chilliard Y., 2005b. Effects of ruminal or duodenal supply of fish oil on milk fat secretion and profiles of trans-fatty acids and conjugated linoleic acid isomers in dairy cows fed maize silage. Anim. Fd. Sci. Tech., 119, $227-$ 246 .

Lucas A., Agabriel C., Martin B., Ferlay A., Verdier-Metz I., Coulon J.B., Rock E., 2006. Relationships between the conditions of cow's milk production and the contents of components of nutritional interest in raw milk farmhouse cheese. Lait, 86, 177-202.

Mourot J., 2004. Effets d'une alimentation enrichie en Omega 3 sur la qualité nutritionnelle des viandes. NAFAS, 2, 30-33.

Mourot J., Blochet J.E., Kouba M., 2006. Effet des acides gras n-3 sur la qualité nutritionnelle de la viande de lapin. Viandes et Produits Carnés, Hors série, 89-90.

Mourot J., Guillevic M., Fillaut M., Robin G 2008. Effet de l'incorporation d'huile de chanvre dans le régime sur les performances de croissance et la qualité nutritionnelle de la viande porc. 40 èmes Journ. Rech. Porcine Fr., sous presse.

Office de l'Elevage, 2006. Marché des produits carnés d'origine bovine. In : Le marché des produits carnés, avicoles et laitiers : chiffres clés 2006. Publications de l'Office de l'Elevage, 11-25.

Palmquist DL, Beaulieu AD, Barbano DM., 1993. Feed and animal factors influencing milk fat composition. J. Dairy Sci., 76, 1753-1771.

Roy A., Ferlay A., Shingfield K.J., Chilliard Y., 2006. Examination of the persistency of milk fatty acid composition responses to plant oils in cows given different basal diets, with particular emphasis on trans-C18:1 fatty acids and isomers of conjugated linoleic acid. Anim. Sci., 82, 479492 .

Roy A., Chardigny J.M., Bauchart D., Ferlay A., Lorenz S., Durand D., Gruffat D., Faulconnier Y., Sébédio J.L., Chilliard Y., 2007. Butters rich either in trans-10-C18:1 or in trans -11-C18:1 plus cis-9, trans-11 CLA differentially affect plasma lipids and aortic fatty streak in experimental atherosclerosis in rabbits. Animal, 1, 467-476.

Rymer C., Givens D.I., 2005. n-3 Fatty acid enrichment of edible tissue of poultry: a review. Lipids, 40, 121-130.

Schmidely P., Sauvant D., 2001. Taux butyreux et composition de la matière grasse du lait chez les petits ruminants : effets de l'apport de matières grasses ou d'aliment concentré. INRA Prod. Anim., 14, 337-354.

Scollan N.D., Richardson I., De Smet S., Moloney A.P., Doreau M., Bauchart D., Nuernberg K., 2005. Enhancing the content of beneficial fatty acids in beef and consequences for meat quality. In: Indicators of milk and beef quality, EAAP, Academic Publishers (Ed), Wageningen, Netherlands, 112, 151-162.

Shingfield K.J., Chilliard Y., Toivonen V., Kairenius P., Givens D.I., 2008. Trans fatty acids and bioactive lipids in ruminant milk. Adv. Exp. Med. Biol., 606, 3-65.

Toullec R., Bauchart D., Bertrand G. Hocquette J.F., Meschy F., 2003. Les aliments d'allaitement. In : Le veau de boucherie, concilier bien-être animal et production. I. Veissier, G. Bertrand, R. Toullec (Eds), INRA Editions, Paris, France, 55-92.

Van Elswyk M.E., 1997. Comparison of n-3 fatty acid sources in laying hen rations for improvement of whole egg nutritional quality: a review. Br. J. Nutr., 78, S61-S69.

Viau M., Gandemer G., Meunier F., Lessire M., Juin H., 1999 Lipides alimentaires et qualité de la viande de poulet : influence de l'apport de quantité croissante d'acide linoléique (18:2 n-6). 3 èmes Journ. Rech. Avicole, 23-25 Mars, SaintMalo, France, 259-362.

Volatier J.L., 2000. Apports en énergie et nutriments pour les adultes et les enfants. In : Enquête individuelle et nationale sur les consommations alimentaires, Tec et Doc, Lavoisier (Ed), Paris, France, 101-119.

Wahle K.W.J., Heys S.D., Rotondo D., 2004. Conjugated linoleic acids: are they beneficial or detrimental to health. Prog. Lipid Res., 43, 553587.

Weill P., Schmitt B., Chesneau G., Daniel N., Safraou F., Legrand P., 2002. Effects of introducing linseed in livestock diet on blood fatty acid composition of consumers of animals products. Ann. Nutr. Metab., 46, 182-191.

Wood J.D., Enser M., Fisher A.V., Nute G.R. Sheard P.R., Richardson R.I., Hughes S.I., Whittington F.M., 2007. Fat deposition, fatty acid composition and meat quality: a review Meat Sci., sous presse.

\section{Résumé}

La composition en Acides Gras (AG) du lait et de la viande est une composante importante de leur qualité nutritionnelle pour l'Homme, qui est fortement modulable à court terme par l'alimentation des animaux d'élevage. Cet article résume les grands axes de recherche et les principaux résultats obtenus à l'INRA sur ce sujet au cours des 10 dernières années. Chez le porc, le poulet et le lapin, les recherches ont principalement porté sur le transfert des AG polyinsaturés, en particulier le 18:3 n-3 du lin, de l'aliment à la viande (muscle et tissu adipeux) et ses conséquences sur la qualité des carcasses. Chez les ruminants, on a étudié les effets des principaux types de fourrages (herbe pâturée, foins, ensilages d'herbe ou de maïs) et de concentrés (céréales, oléagineux), et leurs interactions, sur les AG du lait et de la viande bovine, notamment les AG saturés et insaturés (oléique, trans, conjugués et 18:3 n-3). Des différences marquées existent entre les réponses des AG du lait de la vache et de la chèvre laitière. Des études en cours évaluent les éventuels effets secondaires des pratiques alimentaires sur la qualité sensorielle des produits et la santé des animaux, et sur une possible utilisation du profil en AG pour tracer l'origine des produits. Les études futures devront aussi intégrer l'avancée attendue des connaissances en nutrition humaine sur les effets potentiels des différents AG majeurs et mineurs des produits animaux standards ou enrichis. 


\begin{abstract}
Product quality: modulation of milk and meat fatty acid composition by animal feeding

Milk and meat fatty acid (FA) composition is an important component of their nutritional quality for human consumption. This composition can be markedly and rapidly modulated by feeding factors in farm animals. This paper summarizes the main research directions and results obtained by INRA on these topics during the last 10 years. In the pig, chicken and rabbit, research was focused mainly on the transfer of polyunsaturated FA, particularly 18:3 n-3 from linseed, from feed to meat (muscle and adipose tissue) and its consequences on carcass quality. In ruminants, studies were on the effects of the main forage types (pasture, hays, grass or maize silages) and concentrates (cereals, oilseeds), and their interactions, on bovine milk and meat FA, particularly saturated and unsaturated FA (oleic, trans, conjugated and 18:3 n-3). Marked differences were observed between the dairy cow and goat milk FA responses. Current studies are being done to assess putative secondary effects of feeding practices on product sensory quality and animal health, as well as the potential of using FA profile to trace animal product origin. Future studies should also take into account expected progress in human nutrition knowledge on putative effects of major and minor FA, which are present in standard or enriched animal products.
\end{abstract}

CHILLIARD Y., BAUCHART D., LESSIRE M., SCHMIDELY P., MOUROT J., 2008. Qualité des produits : modulation par l'alimentation des animaux de la composition en acides gras du lait et de la viande. INRA Prod. Anim., 21, 95-106. 
